
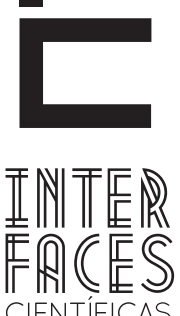

CIENTÍFICAS

HUMANASE SOCIAIS

\title{
PERFIL AGLOMERATIVO \\ DA INDÚSTRIA EXTRATIVA MINERAL EM SERGIPE
}

Reilane Gasparoni da Silva²

\section{RESUMO}

O estado de Sergipe está tem sido caracterizado por um nova dinâmica de crescimento de seus indicadores que estão diretamente relacionados com os recursos naturais existentes em seu subsolo. No entanto, esse processo tem se restringido a espaços específicos (sub-regiões), que se destacam em termos dos níveis de emprego e renda superiores a média do conjunto do Estado. Por isso o presente artigo visa analisar por meio da análise multivariada de agrupamento identificar o perfil aglomerativo da indústria extrativa mineral segundo as microrregiões sergipanas. A análise de agrupamento será realizada com base em dados secundários, para o ano de 2010, utilizando informa- ções provenientes do banco de dados da Relação Anual de Informações Sociais (RAIS/MTE) e do Banco de Dados Agregado (IBGE). As variáveis analisadas serão o número de estabelecimentos e trabalhadores das atividades extrativas minerais; produto interno bruto e per capita segundo as microrregiões; valor adicionado, participação no valor adicionado e taxa de crescimento do valor adicionado da indústria.

\section{PALAVRAS-CHAVE}

Concentração Industrial. Recursos Naturais. Crescimento Econômico de Sergipe. Análise de Agrupamentos. 


\section{ABSTRACT}

The State of Sergipe has been characterized by a new growth dynamic of their indicators that are directly related to the natural resources that exist in your basement. However, this process has been restricted to specific areas (subregions), which stand out in terms of employment levels and income above the average of the whole State. So this article aims to analyze through cluster analysis to identify the profile agglomerative mining industry according to micro-regions of Sergipe. The cluster analysis will be based on secondary data for the year of 2010 using information from the database of the Annual Report of Social Information (RAIS/MTE) and Aggregated Database (IBGE). The variables analyzed are the number of establishments and workers in mineral extraction activity; gross domestic product and per capita according to the micro-regions; value added, participation in value added and growth rate of value added in industry.

\section{KEYWORDS}

Industrial Concentration. Natural Resources. Economic Growth of Sergipe. Cluster Analysis.

\section{INTRODUÇ̄̃O}

A dinâmica regional a partir das transformações da economia internacional e da orientação liberalizante da política econômica brasileira, particularmente a partir da década de 1990, passa a se caracterizar pela maior diferenciação inter-regional e por um processo de fragmentação concentradora da produção industrial. Nesse novo cenário podemos visualizar a dinâmica intra-regional do estado de Sergipe. 0 artigo buscar-se-á analisar as atividades da indústria extrativa mineral em Sergipe, via recorte microrregional,

\section{RESUMEN}

El estado de Sergipe es caracterizado por una nueva dinámica de crecimiento de los indicadores que están directamente relacionados con los recursos naturales existentes en su sótano. Sin embargo, este proceso ha sido restringido a espacios específicos (subregiones), que se destacan en términos de empleo y niveles de ingresos más altos que el promedio de todo el estado. Por eso, este artículo tiene como objetivo el análisis mediante la agrupación de variaciones diversas, identificar el perfil de la industria de extracción de minerales de aglomeración según microregiones sergipanas. El análisis de agrupamiento se basará en datos secundarios, para el año 2010, usando información de la base de datos anual de información social (RAIS/MTE) y base de datos agregados (IBGE). Las variables analizadas son el número de establecimientos y trabajadores de la actividad extractiva de mineral; producto interno bruto y per cápita según las microregiones; valor agregado, la participación en el valor añadido y el valor añadido a la tasa de crecimiento de la industria.

\section{PALABRAS CLAVE}

Concentración Industrial. Recursos Naturales. Crecimiento Económico de Sergipe. Análisis de Agrupamiento.

com objetivo é confirmar por meio de análise de agrupamento as concentrações desse setor no Estado.

O trabalho está subdividido em três partes. 0 primeiro capítulo traz uma revisão da literatura acerca do contexto regional brasileiro. Em seguida faz-se uma introdução da trajetória industrial do Nordeste e do estado de Sergipe. E o terceiro capítulo aplica-se a análise de agrupamentos a fim de identificar as aglomerações da atividade extrativa mineral segundo as microrregiões sergipanas. 


\section{CONTEXTUALIZANDO A DINÂMICA REGIONAL BRASILEIRA}

A consolidação do mercado interno nacional na segunda metade do Século XX promoveu a integração produtiva entre as regiões do país. Segundo Guimarães Neto (1989), quando a articulação econômica do capital produtivo de uma região emigra para outras regiões, esse processo implica, necessariamente, na redefinição de novas formas de reprodução do capital ou da força de trabalho. Segundo Cano (1997), o crescimento da região central impulsionava o crescimento das regiões periféricas, seu argumento parte da noção que o crescimento da economia paulista - 0 núcleo da acumulação produtiva do país - implicava em crescimentos regionais diferenciados aos seus complementos espaciais. E ressalta que apesar do caráter concentrador que a dinâmica de acumulação assume, seus resultados concretos articulam o crescimento regional.

Entre 1930 e 1970, o país apresentou uma das maiores taxas de crescimento econômico do mundo capitalista, em termos internos. O período 1970 a 1985 caracterizou-se pela fase de implantação da matriz industrial, neste sentido a acumulação exigia maior articulação, especificamente da base de recursos naturais. Isso implicou uma maior articulação entre a região central e regiões periféricas, especificamente as regiões com melhor base de recursos naturais. Por outro lado, os investimentos regionalizados em indústrias leves e de insumo, impulsionados pelas políticas de desenvolvimento regionais implantadas a partir de meados da década 60, promoveram o processo de desconcentração industrial inter-regional (CANO, 1997).

O processo de integração dos anos 1960-1980 redefiniu as bases da questão regional no país, porém não foi capaz de avançar o suficiente com vistas a reduzir as desigualdades inter-regionais. Como elemento agravante, a longa crise da economia brasileira que se prolongou dos 1980 até meados dos anos 1990 vai impor sérias restrições ao desenvolvimento regional no país. Nessa perspectiva, Pacheco (1996) argumenta que a década de 1990 rompeu, em certo sentido, a lógica de integração do mercado nacional - tema econômico de promoção do desenvolvimento regional do período anterior. Dito de outro modo, o contexto de crise e estagnação pós 1980 caracterizou um novo contexto a cerca dos temas econômicos regionais, a saber, a natureza fragmentada e espacialmente heterogênea do desenvolvimento econômico.

Segundo Araújo (1999), o rompimento do papel ativo que o Estado nacional desempenhava nas décadas anteriores, tanto por políticas explicitamente regionais como por políticas de corte setorial-nacional, além do papel expressivo que as empresas estatais exerciam na dinâmica dos espaços. Neste contexto, as decisões de investimento tendem a se limitar ao setor privado, dado a crise do Estado e as novas orientações governamentais, associados à indefinição e atomização que têm marcado a política de desenvolvimento regional no país. E argumenta que a nova política regional deve está fundamentada na herança heterogênea que caracteriza a realidade espacial do país, com regiões e sub-regiões desenvolvidas e industrializadas, ao lado de regiões e sub-regiões atrasadas. A autora ressalta que a atuação de um mercado auto-regulado, num quadro de globalização e inserção competitiva tende a privilegiar os espaços integrados aos fluxos econômicos internacionais, por outro lado tendem a marginalizar as áreas menos dinâmicas, elevando os custos sociais.

Pode-se concluir que a integração econômica que articulou as diversas dinâmicas regionais não homogeneizou as estruturas produtivas, permanecendo diferenciações importantes entre as regiões do país. No contexto atual, dinâmica regional está submetida a um conjunto de fatores que atuam tanto no sentido 
de acelerar ou reduzir as taxas de crescimento específicas dos espaços econômicos. 0 contexto macroeconômico nacional e internacional, a dimensão institucional, a dinâmica populacional passar a ter cada vez mais influência na dinâmica das economias regionais.
Nesta perspectiva o próximo capitulo trará as trajetórias do desenvolvimento industrial do estado de Sergipe que parece ser moldado por esse contexto tratado neste capítulo.

\section{TRAJETÓRIAS DO DESENVOLVIMENTO INDUS- TRIAL DO ESTADO DE SERGIPE}

O crescimento industrial e a desconcentração relativa decorreram da expansão das industriais básicas, articuladas a base de recursos naturais e dos bens de consumo duráveis (DINIZ, 1995). A região Nordeste iniciou esse processo na década de 1960 em decorrência de um intenso fluxo de capitais de empresas do Centro-Sul em direção à região, resultando numa radical modificação da sua estrutura produtiva, com a implantação de unidades industriais modernas, tecnologicamente mais avançadas e em setores novos (MELO et al, 2009).

A ausência do Estado Nacional cedeu lugar as esferas subnacionais (Estados e Municípios) no sen-

\subsection{CARACTERIZAÇ̃̃O DA PRODUCC̄̃̃ EM SERGIPE}

Em Sergipe, como na maioria dos estados nordestinos, o contexto de criação da SUDENE proporcionou mudanças significativas que transformaram a estrutura produtiva do estado, que até então, era pouco diversificada com predomínio no setor rural - da cana-de-açúcar, algodão e pecuária - complementada pelas culturas de subsistência. A economia sergipana diferencia-se da maioria dos demais estados nordestinos pela riqueza em seu subsolo o que proporciona ao estado novas oportunidades de exploração de recursos minerais (MELO et al, 2009). tido de desempenharem a função de novo agente na definição e implementação de políticas e programas governamentais, alicerçados nas teorias de desenvolvimento local. Com base nesse novo contexto, os agentes subnacionais passaram a praticar disputas por investimentos produtivos, como alternativas, na ausência de políticas de desenvolvimento regional (FEITOSA, 2007). Na visão de Araújo (1995) as subáreas com baixo grau de dinamismo resultam, em parte, pela ausência explícita de políticas regionais por parte do governo federal. Lacuna que deflagra a guerra fiscal entre estados e municípios, que buscam contribuir para consolidar focos de dinamismos em suas áreas de atuação.

Durante a expansão da economia brasileira nos anos 1960 e 1970, a economia nordestina experimentou mudanças estruturais significativas, decorrentes principalmente das novas formas de atuação do Estado, que se traduziram no crescimento econômico e no estabelecimento de uma nova divisão territorial do trabalho. A região Nordeste passou a se especializar, cada vez mais, na produção de bens intermediários como forma de suprir as demandas do mercado nacional, conformando o que se convencionou chamar de "nova indústria". Este processo de "integração produtiva" e o surgimento da "nova indústria" no estado 
de Sergipe somente vieram ocorrer a partir de meados da década de 1970, quando boa parte dos demais estados nordestinos já apresentava estruturas produtivas com características distintas daquelas verificadas antes das políticas de desenvolvimento regional da SUDENE (FEITOSA, 2007).

Apesar dessa defasagem temporal, o estado de Sergipe, de modo particular, vem se destacando nas últimas décadas, por apresentar níveis relativamente elevados de crescimento do Valor Adicionado industrial (VA), levando em conta o tamanho desse espaço e os padrões de desenvolvimento da região Nordeste.
Os determinantes que inserem o Estado de Sergipe nessa nova dinâmica de crescimento e melhora em seus indicadores estão relacionados com vantagens competitivas, que vão desde os recursos naturais existentes, localização geográfica, tamanho e nível de renda da população. No entanto, esse processo tem se restringido a espaços específicos (sub-regiões), que se destacam em termos dos níveis de emprego e renda superiores a média do conjunto do Estado. Nesta perspectiva, o trabalho utiliza como ferramenta de análise o recorte microrregional adotado pelo Instituto Brasileiro Geografia e Estatística (IBGE) a fim de identificar as microrregiões que se destacam no segmento industrial, particularmente das atividades extrativa mineral do Estado.

TABELA 1 - SERGIPE - Participação Relativa e Taxa Média de Crescimento do Valor Adicionado Industrial a preços básicos segundo as microrregiões, 2001-2010.

\begin{tabular}{|c|c|c|c|}
\hline \multirow{2}{*}{ Microrregiões } & \multicolumn{2}{|c|}{ Participação (\%) } & \multirow{2}{*}{$\begin{array}{c}\text { Taxa Média de Crescimento (\%) } \\
2001-2010 \\
\end{array}$} \\
\hline & 2001 & 2010 & \\
\hline SERGIPE & 100,0 & 100,0 & 4,5 \\
\hline Sergipana do Sertão do São Francisco & 24,4 & 20,5 & 6,3 \\
\hline Carira & 1,5 & 2,3 & 7,3 \\
\hline Nossa Senhora das Dores & 0,5 & 0,7 & 7,6 \\
\hline Agreste de Itabaiana & 1,8 & 2,2 & 5,3 \\
\hline Tobias Barreto & 1,2 & 1,5 & 4,8 \\
\hline Agreste de Lagarto & 2,2 & 1,8 & 2,0 \\
\hline Propriá & 2,3 & 1,7 & 1,2 \\
\hline Cotinguiba & 1,6 & 4,8 & 18,5 \\
\hline Japaratuba & 4,3 & 5,0 & 6,1 \\
\hline Baixo Cotinguiba & 10,2 & 16,3 & 9,9 \\
\hline Aracaju & 37,3 & 32,1 & 1,7 \\
\hline Boquim & 1,2 & 1,6 & 5,1 \\
\hline Estância & 11,6 & 9,5 & 0,7 \\
\hline
\end{tabular}

Fonte: IBGE (2012). Cálculos e Elaboração Própria.

Nota: Para efeitos dos cálculos da taxa média de crescimento foram utilizados a média móvel bienal, tomando o ano anterior e o ano corrente do período 2000-2010. 
A Tabela 1 revela, por meio da participação do VA industrial, a heterogeneidade inter-regional no estado de Sergipe. Constata-se a existência de diferenciações entre as microrregiões sergipanas, com cinco das treze microrregiões apresentando estruturas industriais relativamente mais importantes do ponto de vista estadual do que as demais. Dentre as treze microrregiões, seis participam com $86,2 \%$ do VA industrial estadual (em 2010). Destaque para as microrregiões de Aracaju (32,1\%), Sergipana do Sertão do São Francisco $(20,5 \%)$ e Baixo Cotinguiba (16,3\%).

Em termos de taxa média de crescimento, pode-se verificar que no período de 2001 a 2010 todas as microrregiões obtiveram taxas de crescimentos signifi- cativas destaque para Contiguiba (18,5\%) e Baixo Cotinguiba $(9,9 \%)$. Em alguns casos o baixo crescimento deve-se à base forte de alguns espaços em termos de VA industrial, cujo incremento precisa ser bastante elevado para que seja demonstrado na taxa média de crescimento. Como é o caso da microrregião de Aracaju (Tabela 1).

Conforme demonstrado na Tabela 2, no ano de 2001 a indústria extrativa representava 3,9\% do incremento do valor adicionado no estado. Em 2010 houve incremento de quase dois pontos no percentual. Enquanto a indústria de transformação segue com perdas de mais de quatro pontos no mesmo período.

TABELA 2 - SERGIPE - Participação da Indústria no Valor Adicionado Bruto a preços básicos, 2001-2010.

\begin{tabular}{|l|c|c|}
\hline \multicolumn{1}{|c|}{ INDÚSTRIA DE SERGIPE } & \multicolumn{2}{c|}{ Participação } \\
\cline { 2 - 3 } \cline { 2 - 3 } & $\mathbf{2 0 0 1}$ & $\mathbf{2 0 1 0}$ \\
\hline Indústria Extrativa & 3,9 & 5,8 \\
\hline Indústria Transformação & 11,8 & 7,4 \\
\hline Produção e Distribuição de Eletricidade e Gás, Água, Esgoto e Limpeza Urbana. & 9,2 & 7,8 \\
\hline Construção Civil & 6,2 & 7,6 \\
\hline SERGIPE & 31,2 & 28,6 \\
\hline
\end{tabular}

Fonte: IBGE (2012).

Dado que a ênfase desse trabalho está voltada para a base extrativa mineral a partir do recorte microrregional, buscar-se-á no próximo capítulo confirmar quais são as microrregiões que possuem como base de produção as atividades extrativas minerais.

Com base na matriz de dados da Rais verificou-se por meio do número de estabelecimentos e número de trabalhadores nas atividades extrativas minerais a representatividade de algumas microrregiões, particularmente as microrregiões de Cotinguiba, Bai- xo Cotinguiba, Japaratuba e Aracaju. A fim de confirmar esses dados se fará o uso da análise de agrupamento para o ano de 2010. 


\section{ANÁLISE DE AGRUPAMENTO}

A análise de agrupamentos ou clusters é uma técnica multivariada que agrega objetos com base nas características que eles possuem, classificando objetos de modo que cada um seja semelhante aos outros no agrupamento em relação a algum critério de seleção predeterminado. Os agrupamentos resultantes de objetos devem exibir elevada homogeneidade interna (dentro dos agrupamentos) e elevada heterogeneidade externa (entre agrupamentos). É caracterizada por uma análise descritiva sem base teórica e subdividese em duas categorias - hierárquica e não-hierárquica. 0 método hierárquico está baseado numa construção de estrutura do tipo árvore, pode ser do tipo aglomerativo ou divisivo (HAIR et al, 2005).

Nos métodos aglomerativos, cada objeto ou observação começa como seu próprio agrupamento no final todos os objetos são reunidos em um grande agregado. Quando o processo de agrupamento prossegue na direção oposta dos métodos aglomerativos denominase de método divisivo. Esse método começa com grande agregado que contém todos os objetos, o processo continua até que cada objeto seja um agrupamento por si mesmo (HAIR et al, 2005).

Segundo Hair e outros (2005) existem cinco algoritmos que são utilizados para desenvolver agregados, a saber: ligação individual, ligação completa, ligação média, método de Ward e método de centroíde. Esses algoritmos diferem na forma como a distância entre os agrupamentos é computada. No caso da Ligação individual o procedimento é baseado na distância mínima, também chamado de vizinho mais próximo. A distância entre dois agrupamentos quaisquer é a menor distância de qualquer ponto de um agregado até qualquer ponto do outro. A Ligação completa é semelhante ao da ligação individual, porém o critério de agrupamento se baseia em distância máxima. A Ligação média começa da mesma forma que a ligação individual/completa, mas o critério de agrupamento é a distância média de todos os indivíduos em um agru- pamento aos demais em outro. No Método de Ward, a distância entre dois agrupamentos é a soma dos quadrados entre os dois agrupamentos feita sobre todas as variáveis, esse procedimento tende a combinar agrupamentos com um pequeno número de observações. E por fim, o Método centróide que está baseado na distância entre dois agrupamentos, isto é, na distância entre seus centroides.

No caso do método não-hierárquico, o processo de agrupamento é mais dinâmico e interativo, uma vez que os objetos se agrupam simultaneamente. 0 critério não-hierárquico mais utilizado é o K-means, que permite a definição do vetor central dos clusters e insere os objetos mais próximo. Com isso se estabelece o número de clusters e testa-se a hipótese segundo o significado encontrado para cada solução (GONG; RICHMAN, 1995).

Há vários trabalhos que se utilizarão da análise de agrupamento ou clusters dentre eles pode-se destacar Simões e demais autores (2004) cujo o seu trabalho utilizou a análise de cluster para identificar a rede urbana de oferta de serviços de saúde a nível municipal para o estado de Minas Gerais. Outro trabalho realizado por Silva e Silva e Simões (2004) utilizou a análise de clusters para identificar oportunidades tecnológicas a partir de indicadores de produção científica e emprego industrial em nível microrregional no Brasil.

Os conceitos de distância são importantes para a compreensão da análise de agrupamento. Este trabalho parte do método de agrupamento aglomerativo, utilizando o algoritmo do vizinho mais próximo (ligação individual) com base na metodologia da distância euclidiana quadrática. Com o objetivo de agrupar as microrregiões do Estado de Sergipe em termos dos escores dos indicadores de número de estabelecimentos da indústria extrativa mineral, número de trabalhadores da indústria extrativa mineral, PIB das microrre- 
giões, PIB per capita, valor adicionado da indústria, participação do valor adicionado da indústria e taxa de crescimento do valor adicionado da indústria. As variáveis foram selecionadas com base na disponibilidade de variáveis desagregadas para os municípios sergipanos tentou-se buscar as variáveis que pudessem representar o agrupamento da base extrativa mineral. Já que o agrupamento é feito de forma que haja homogeneidade intragrupos e heterogeneidade intergrupos.

A análise de agrupamento será realizada com base em dados secundários, para o ano de 2010, utilizando informações provenientes do banco de dados da Relação Anual de Informações Sociais (RAIS/MTE) e do Banco de Dados Agregado (IBGE). As variáveis analisadas serão o número de estabelecimentos e trabalhadores das atividades extrativas minerais; produto interno bruto e per capita segundo as microrregiões; valor adicionado, participação no valor adicionado e taxa de crescimento do valor adicionado da indústria. Sendo que as variáveis de valor adicionado da indústria e número de trabalhadores da indústria extrativa mineral assumirá o papel de variável preditiva que servirá de base para validação do agrupamento. 0 tópico a seguir apresentará os resultados dessa análise.

\subsection{ANÁLISE E RESULTADOS}

No intuito de classificar as microrregiões de Sergipe em agrupamento das atividades relacionadas com a indústria extrativa mineral através do agrupamento de escores de acordo com os indicadores de número de estabelecimentos da indústria extrativa mineral, PIB das microrregiões, PIB per capita, participação do valor adicionado da indústria e taxa de crescimento do valor adicionado da indústria. Utilizou-se a análise multivariada com o método de agrupamento (também conhecida na literatura como análise de cluster) cuja metodologia adotada foi o método hierárquico de ligação individual que agrupou as microrregiões com as menores distâncias (segundo os indicadores utilizados) primeiramente, e em seguida foram formados maiores cadeias agrupando todos os indivíduos em uma única.

Conforme pode ser visualizado na Figura 1 no lado esquerdo têm-se o agrupamento formado e do lado direito está a validação do agrupamento que foi realizado por meio de duas variáveis preditivas o número de trabalhadores da indústria extrativa mineral e valor adicionado da indústria. No lado esquerdo os primeiros agrupamentos (na base) possuem combinações indesejáveis que são captadas por alguns indicadores.
Para fins de agrupamentos da indústria extrativa percebe-se a presença de três grupos o primeiro formado por Japaratuba que está unido as demais microrregiões com algum grau de indústria presente nestes municípios, não necessariamente, do setor extrativo; o segundo grupo verifica-se a presença da microrregião de Cotinguiba e da microrregião de Baixo Cotinguiba e o terceiro que pode ser caracterizado como um outlier, mas que é típico devido ao impacto da capital do estado presente nesta microrregião (11) onde predomina o maior diferencial dos indicadores estudados para todo o estado de Sergipe. Portanto a outlier Aracaju representa uma componente estrutural válida.

No lado esquerdo foram utilizadas duas variáveis preditivas como método de validação do agrupamento das microrregiões como pode ser visualizado tem-se a confirmação da microrregião de Japaratuba, Baixo Cotinguiba e Aracaju como os três maiores agrupamentos o que destoou foi à microrregião de Cotinguiba, mas isso se deve a qualidade dos dados desagregados que são raros de ser encontrados no Estado, então quando foi adotado o número de trabalhadores e estabelecimentos para 
captar a localização tem-se um resultado por vezes viezado devido a dinâmica do próprio setor de extração mineral que comporta um reduzido número de trabalhadores bem como de estabelecimento, porém agrega valor adicionado industrial para 0 estado de forma significativa. Entretanto, não foi possível encontrar o valor adicionado da indústria extrativa para o ano de 2010 desagregado, isto é, para os municípios a fim de agrupa-los e nem dados desse indicador no recorte microrregional para o mesmo período.
Como forma de teste utilizou-se a análise de ajustamento por meio do teste não paramétrico de Shapiro -Wilk, baseado na estatística W. É um teste padrão para verificar a normalidade de uma amostra. A estatística do teste pode ser pensada como a correlação entre os dados e os valores correspondentes da normal. Se W for igual a 1, então a distribuição dos dados é perfeitamente normal. Quando W é significamente menor do que 1, a suposição de normalidade não é atendida. 0 teste Shapiro-Wilk é recomendado para amostras pequenas ${ }^{1}$.

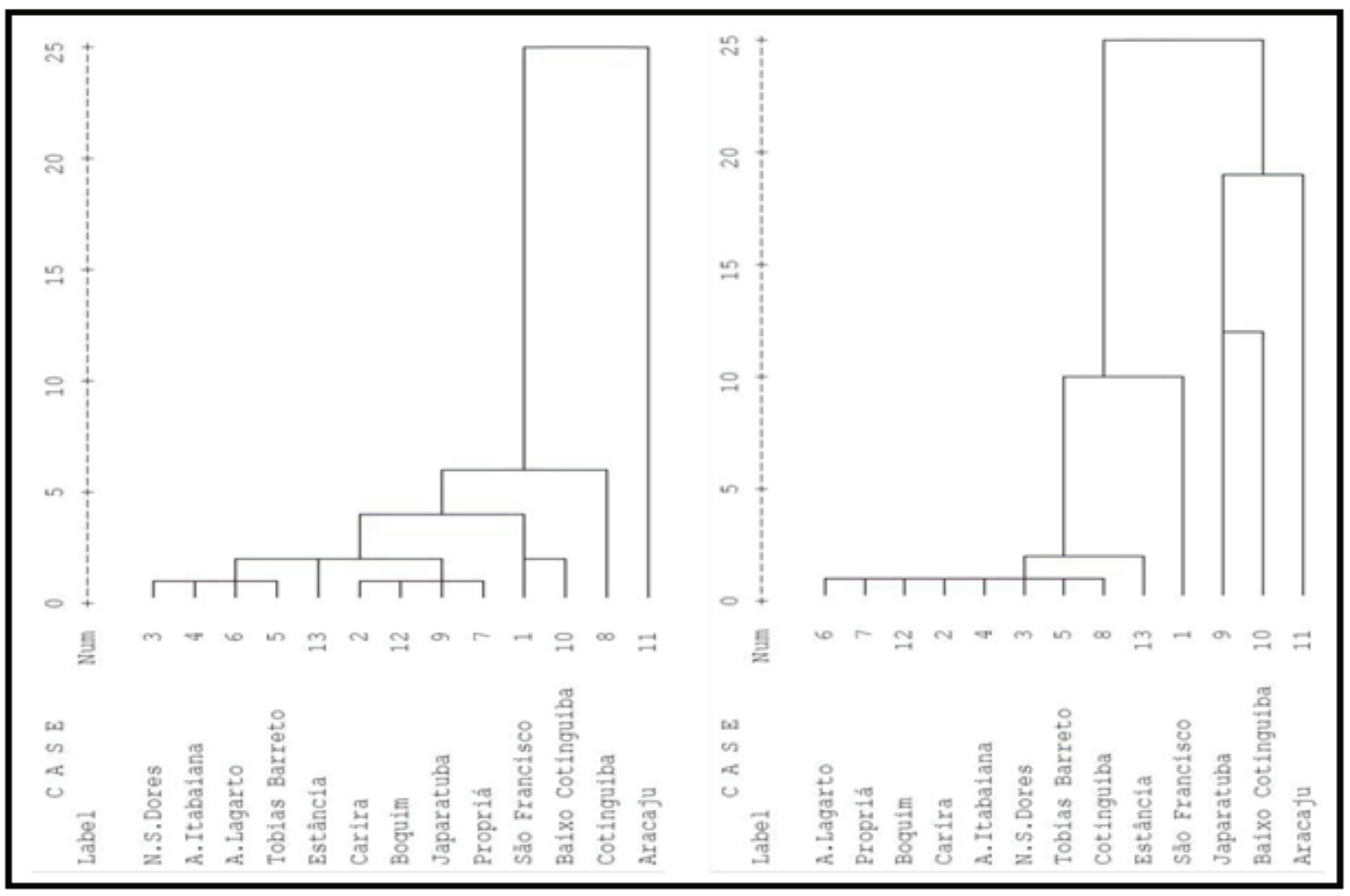

Fonte: Elaboração Própria com utilização do software SPSS.

FIGURA 1 -Agrupamentos Extrativos Minerais segundo microrregiões de Sergipe, 2010.

1 Para maiores informações ver SHAPIRO e WILK (1965). Neste trabalho o cálculo do teste $W$ foi realizado com o software SPSS. 


\begin{tabular}{|c|c|c|c|}
\hline & \multicolumn{3}{|c|}{ Shapiro-Wilk } \\
\hline & Statistic & df & Sig. \\
\hline $\begin{array}{l}\text { No estabelecimentos das } \\
\text { microrregiōes na } \\
\text { indústria extrativa mineral }\end{array}$ & ,581 & 13 & ,OOO \\
\hline PIB das microrregiöes & ,480 & 13 & ,OOO \\
\hline $\begin{array}{l}\text { PIB per capita das } \\
\text { microrregiōes }\end{array}$ &, 907 & 13 &, 164 \\
\hline $\begin{array}{l}\text { Valor adicionado da } \\
\text { indústria nas } \\
\text { microrregiōes }\end{array}$ & ,735 & 13 & ,OO1 \\
\hline $\begin{array}{l}\text { Participação das } \\
\text { microrregiōes no valor } \\
\text { adicionado da indústria }\end{array}$ &, 734 & 13 & ,001 \\
\hline $\begin{array}{l}\mathrm{N}^{\circ} \text { trabalhadores das } \\
\text { microrregiōes na } \\
\text { indústria extrativa mineral }\end{array}$ &, 585 & 13 & ,OOO \\
\hline $\begin{array}{l}\text { Taxa de crescimento das } \\
\text { microrregiōes no valor } \\
\text { adicionado da indústria }\end{array}$ & ,850 & 13 & ,028 \\
\hline
\end{tabular}

Fonte: Elaboração Própria com utilização do software SPSS.

\section{FIGURA 2-Resultado do Teste SHAPIRO-WILK}

Para testar as hipóteses foi denominado $\mathrm{H}_{0}$ quando os dados da amostra seguirem uma distribuição normal; e, $\mathrm{H}_{1}$ para a situação em que os dados da amostra não seguem uma distribuição normal. Tendo-se a decisão de rejeitar $\mathrm{H}_{0}$ ao nível de significância $\mathrm{a}=0,05$ se o $W_{\text {calculado }} W_{\text {crítico: }}$ A Figura 2 apresenta a estatística de Shapiro-Wilk (W) para os dados utilizados na análise de agrupamento das microrregiões sergipanas.

O cálculo da estatística $W$ demonstrou para as sete características estudadas que as variáveis números de estabelecimento na indústria extrativa mineral, PIB, Valor adicionado da indústria, participação das microrregiões no valor adicionado da indústria, número de trabalhadores na indústria extrativa mineral e taxa de crescimento do valor adicionado da indústria tiveram o $W_{\text {calculado }}$ menor do que o $W_{\text {crítico }}$, isto é, deve-se rejeitar a hipótese nula $\left(\mathrm{H}_{0}\right)$ subentendendo-se que os dados não seguiram uma distribuição normal. Exceto o PIB per capita que obteve $\mathrm{W}_{\text {calculado }}=$ $0,907 W_{\text {crítico }}=0.866$. Nesta situação se aceita a hipótese de que a amostra tenha uma distribuição normal (FIGURA 2). Os resultados estatísticos só confirmaram o que os dados em valores absolutos já expressam. Ou seja, que há uma distribuição normal entre as microrregiões, isto é, existe heterogeneidade bastante expressiva em termos de uma base industrial, particularmente das indústrias extrativas minerais. 


\section{CONSIDERACÕ̃ES FINAIS}

A dinâmica regional está submetida a um conjunto de fatores que atuam tanto no sentido de acelerar ou reduzir as taxas de crescimento específicas dos espaços econômicos. Os determinantes que inserem o Estado de Sergipe nessa nova dinâmica de crescimento de seus indicadores estão relacionados, em parte, com os recursos naturais existentes em seu subsolo. No entanto, esse processo tem se restringido a espaços específicos (sub-regiões), que se destacam em termos dos níveis de emprego e renda superiores a média do conjunto do Estado.

Por meio da análise multivariada de agrupamento com o método hierárquico de ligação individual se agrupou as microrregiões com as menores distâncias em relação aos indicadores - número de estabelecimentos e trabalhadores da indústria extrativa mineral; PIB; PIB per capita; valor adicionado da indústria, participação do valor adicionado da indústria e taxa de crescimento do valor adicionado da indústria - a fim de encontrar aglomeração da indústria extrativa mineral conforme os dados brutos dão a entender. Percebe-se que a dinâmica estadual é bastante concentrada espacialmente. A produção industrial está concentrada no entorno da capital do Estado, Aracaju e nos municípios onde há disponibilidade de recursos naturais. As microrregiões que dependem da dotação de fatores não renováveis apresentam pouco grau de encadeamento produtivo. Há um alto grau de concentração e especialização das atividades extrativas minerais.

Os resultados foram confirmados com a presença de três agrupamentos da indústria extrativa mineral. 0 primeiro é formado por Japaratuba que está unido as demais microrregiões que possuem outros tipos de indústria, não necessariamente, do setor extrativo; no segundo grupo verifica-se a microrregião de Cotinguiba e Baixo Cotinguiba e o terceiro grupo que pode ser caracterizado como um outlier, mas que é típico da microrregião de Aracaju. Como teste de hipótese foi utilizado o teste de Shapiro-Wilk que confirmou que os indicadores apresentados para as microrregiões de Sergipe não apresentam normalidades, isto é, existe bastante heterogeneidade em termos da base industrial, especificamente da indústria mineral.

\section{REFERÊNCIAS}

ARAÚJO, Tania Bacelar de (1995) “Nordeste, Nordestes, que Nordeste”? In: AFFONSO, R.B \& SILVA, P.L.B (org.). Desigualdades regionais e desenvolvimento (federalismo no Brasil). São Paulo, Fundap/Unesp, p. 125-156.

ARAÚJO, Tania Bacelar de (1999) "Brasil nos Anos Noventa: Opções Estratégicas e dinâmica regional” Revista Brasileira de Estudos Urbanos e Regionais. Recife: Anpur - Associação Nacional de Pós-Graduação e Pesquisa em Planejamento Urbano e Regional, n.2, p. 9-24, Novembro.

CANO, W. (1997) "Concentração e desconcentração econômica regional no Brasil: 1970/95." Revista Economia e Sociedade, Campinas, Unicamp, n.8, p.101-41, Junho.

DINIZ, Clélio Campolina (1995) “A dinâmica regional recente da economia brasileira e suas perspectivas”. Texto para discussão no 375 . (IPEA), Brasília. 
FEITOSA, Cid Olival As transformações recentes da economia Sergipana: 1970-2005. Campinas, IE/Unicamp. Dissertação de mestrado. 2007.

GONG, X.; RICHMAN, M.B. "On the application to growing season precipitation data in North America East of the rockies”. Journal of Climate, Oklahoma, v.8, p.897-931. 1995.

GUIMARÃES NETO, Leonardo. Introdução à formação econômica do Nordeste. Recife: Editora FUNDAJ/Massangana, 1989.

HAIR JR., J.; ANDERSON, R.; TATHAM, R. e BLACK, W. Análise Multivariada de Dados. 5. ed. Porto Alegre: Bookman, 2005.

IBGE. Valor adicionado da Indústria. Disponível em: http://www.sidra.ibge.gov.br/bda/tabela/listabl. asp?z=t\&c=21, 2012

MELO, R. O. L et al. "Indústria e Desenvolvimento em Sergipe”. Revista Econômica do Nordeste. Fortaleza, v. 40, p. 331-343. 2009.

MTE/RAIS Número de estabelecimentos e empregos industriais. Disponível em: http://sgt.caged.gov.br/XOLAPW.dll/pamLoginMTE?lang=0. Acesso em: julho, 2011.

PACHECO, Carlos Américo. A questão regional brasileira pós 1980: desconcentração econômica e fragmentação da economia nacional. Campinas, Unicamp. Tese de Doutoramento. 1996.

SHAPIRO, S.S.; WILK, M.B. An analysis of variance test for normality (complete samples). Biometrika, v.52, p.591-611. 1965.

SILVA, L. \& SIMÕES, R. Oportunidades tecnológicas e produção científica: uma análise microrregional. Santiago/Chile: Revista EURE, 2004.

SIMÕES, R. et al. Rede urbana na oferta de serviços de saúde em Minas Gerais: uma análise multivariada para 2002. In: Anais... XIV Encontro Nacional de Estudos Populacionais, Caxambu: Abep, 2004. 